\title{
Relación entre habilidades para la resolución de problemas y rendimiento académico en matemáticas de estudiantes universitarios del I ciclo
}

\section{Relationship between problem-solving skills and academic performance in mathematics from university students in the cycle I}

\author{
Ruth Dignidad Solar De La Torre
}

\begin{abstract}
RESUMEN
Objetivo: Identificar el tipo de relación que existe entre la variable desarrollo de habilidades para la resolución de problemas y el rendimiento académico en matemáticas de los estudiantes del I ciclo de la Facultad de Ciencias de la Educación y Humanidades de la Universidad Nacional "San Luis Gonzaga de Ica". Material y Métodos: Se aplicó un diseño descriptivo de tipo correlacional, con una muestra de 42 estudiantes elegidos mediante el azar simple; se utilizó como instrumentos un test de habilidades de resolución de problemas y una prueba de comprobación, y para la prueba de hipótesis se empleó el coeficiente de correlación de Pearson para datos simples. Resultado: La Evaluación entre las variables comprensión del problema (X1) y el promedio de notas obtenidas en las evaluaciones (Y1); entre la capacidad para elaborar un plan (X2) y el nivel de logro de competencias matemáticas (Y2), entre la capacidad para ejecutar y evaluar el plan (X3) y la actitud hacia las matemáticas (Y3), obteniendose en el coeficiente de correlación de Pearson $r=0,837, r=0,830 ; r=0,895$ respectivamente. Conclusión: Existe una relación positiva alta entre el nivel de desarrollo de las habilidades para la solución de problemas y el rendimiento académico en matemática de los estudiantes del primer año de la especialidad de matemática de la Universidad Nacional “San Luis Gonzaga” de Ica.
\end{abstract}

Palabras Clave: Relación positiva, resolución de problemas, rendimiento en matemática.

\section{ABSTRACT}

Objective: To identify the type of relationship that exists between the variable development of problem solving skills and academic performance in mathematics of the students of the I cycle of the Faculty of Education and Humanities Sciences of the National University "San Luis Gonzaga from Ica". Material and Methods: A descriptive design of a correlational type was applied, with a sample of 42 students chosen through simple chance; A problem-solving skills test and a test of evidence were used as instruments, and Pearson's correlation coefficient was used for simple data for the hypothesis test. Result: The evaluation between the variables understanding the problem (X1) and the average of marks obtained in the evaluations (Y1); between the ability to develop a plan (X2) and the level of achievement of mathematical skills (Y2), between the ability to execute and evaluate the plan (X3) and the attitude towards mathematics (Y3), obtaining the correlation coefficient Pearson's $r=0.837, r=0.830 ; r=0.895$ respectively. Conclusion: There is a high positive relationship between the level of development of problem solving skills and the academic performance in mathematics of the first year students of the specialty of mathematics of the National University "San Luis Gonzaga" of Ica.

Keywords: Positive relationship, problem solving, math performance.

\section{INTRODUCCIÓN}

De acuerdo con diversos resultados en los que el Perú ha participado en la evaluación de los aprendizajes de los estudiantes como es el caso de las evaluaciones PISA, el desempeño de los estudiantes no es bueno, más bien ocupamos uno de los últimos lugares de los 64 países participantes en lo relacionado al desarrollo de las habilidades de los estudiantes para la resolución de problemas.

Como señala Ferrer (2000), la resolución de problemas es una de las actividades fundamentales del pensamiento, de modo que permite activar su propia capacidad mental, su capacidad creativa, reflexiva y en general mejorar sus procesos de pensamiento. (p. 23)

Sin embargo en la resolución de problemas donde los estudiantes tienen dificultades en la mayoría de los casos lo cual a su vez está asociado a otros problemas como de la comprensión, dificultades para procesar, analizar, deducir y construir significados.

Si bien es cierto que las evaluaciones PISA han tomado muestras de estudiantes entre 14 y 15 años, en el transcurso de la práctica docente es muy común encontrarse con situaciones en los que aún los estudiantes que inician sus estudios universitarios, siguen mostrando deficiente nivel de desarrollo de las habilidades para la resolución de problemas matemáticos, como es el caso de los estudiantes del primer ciclo de la Facultad de Ciencias de la Educación y
Humanidades de la Universidad nacional San Luis Gonzaga de lca.

Sobre los factores a los que se encuentra asociada esta deficiencia en el desarrollo de las habilidades o capacidades para la resolución de problemas de matemática, existe consenso entre los especialistas de que son muchos. Así, dentro de ellos se puede mencionar desde aquellos factores relacionados con las estrategias o metodologías empleadas por los docentes para posibilitar el desarrollo de las capacidades de resolución de problemas, hasta otros factores de tipo orgánico o biológico que no contribuyen a que los procesos mentales desarrollen la capacidad para la resolución de problemas que por cierto son capacidades complejas y de alta demanda cognitiva.

Asimismo, el desarrollo deficiente de las habilidades para la resolución de problemas, es un factor que condiciona otros problemas como es el caso del rendimiento académico en matemática de los estudiantes.

Tradicionalmente, para una mayoría de estudiantes, la matemática es un curso poco atractivo y no todo es responsabilidad del alumnado, en muchos casos es la inadecuada práctica docente la que no incentiva a los estudiantes; ello principalmente por cuanto las estrategias, recursos y métodos no logran influir positivamente en el desarrollo de aptitudes y actitudes, por el contrario, su fracaso acaba disminuyendo la 
autoestima del alumno.

Es por ello que precisamente se plantea como problema "Relación entre nivel de desarrollo de habilidades para la resolución de problemas y rendimiento académico en el área de matemáticas de los alumnos del primer ciclo de la facultad de educación de la Universidad Nacional "San Luis Gonzaga de Ica" - 2018", de modo que el problema general ha quedado explicitado de la siguiente manera: ¿Qué relación existe entre las habilidades para la resolución de problemas y el rendimiento académico en matemática de los estudiantes del primer año de la especialidad de matemática de la Universidad Nacional "San Luis Gonzaga" de Ica?

En el campo educativo, el enfoque del aprendizaje centrado en la resolución de problemas, como propone Gaulin (2001), tiene muchísima importancia para los estudiantes, ya que les permite a situarse en diversos contextos para crear, recrear, analizar, investigar, plantear y resolver problemas, probar diversos caminos de resolución, analizar estrategias y formas de representación, sistematizar y comunicar nuevos conocimientos, entre otros.

Ahora bien, sobre las estrategias para la enseñanza de la resolución de problemas, según Polya (1945), son 4 fases: Comprender el problema, concebir un plan, ejecutar el plan, examinar la solución obtenida. Cada fase se acompaña de una serie de preguntas cuya intención clara es actuar como guía para la acción. Otros autores como Fustier (1989), proponen 7 etapas.

Entre las habilidades para la resolución de problemas y el rendimiento académico en matemática, existe una relación positiva de modo que cuanto mayor habilidad para la resolución de problemas tienen los estudiantes, mayor o mejor será su rendimiento en matemática. (Batley,1991).

\section{MATERIALES Y MÉTODOS}

Esta investigación, por su finalidad es una investigación descriptiva correlacional, la población se de estudio fue de 83 estudiantes y una muestra de 42 , como técnicas de recolección de datos se empleó la encuesta y la observación y como instrumentos un test de habilidades para la resolución de problemas y una prueba de competencias de matemática, los mismos que fueron validados mediante juicio de expertos. Asimismo el procesamiento de los datos fue de tipo estadístico, empleándose para la prueba de hipótesis el coeficiente de correlación de Pearson para datos sin agrupar.

\section{RESULTADOS}

Los resultados sobre la relación entre las dimensiones de las variables de estudio, expresan básicamente la existencia de una relación positiva, que se evidencia en las siguientes tablas:
Tabla 1

Relación entre habilidades para la comprensión del problema (X1) y promedio de notas obtenidas en las evaluaciones (Y1) de los estudiantes universitarios.

\begin{tabular}{|c|c|c|c|c|c|}
\hline Muestra & $\mathrm{X} 1$ & Y1 & X.Y & $\mathbf{X}^{2}$ & $\mathbf{Y}^{2}$ \\
\hline 1 & 28 & 26 & 728 & 784 & 676 \\
\hline 2 & 33 & 30 & 990 & 1089 & 900 \\
\hline 3 & 25 & 25 & 625 & 625 & 625 \\
\hline 4 & 34 & 39 & 1326 & 1156 & 1521 \\
\hline 5 & 33 & 29 & 957 & 1089 & 841 \\
\hline 6 & 40 & 35 & 1400 & 1600 & 1225 \\
\hline 7 & 38 & 42 & 1596 & 1444 & 1764 \\
\hline 8 & 40 & 42 & 1680 & 1600 & 1764 \\
\hline 9 & 36 & 31 & 1116 & 1296 & 961 \\
\hline 10 & 25 & 30 & 750 & 625 & 900 \\
\hline 11 & 26 & 29 & 754 & 676 & 841 \\
\hline 12 & 34 & 38 & 1292 & 1156 & 1444 \\
\hline 13 & 40 & 43 & 1720 & 1600 & 1849 \\
\hline 14 & 33 & 32 & 1056 & 1089 & 1024 \\
\hline 15 & 24 & 26 & 624 & 576 & 676 \\
\hline 16 & 25 & 23 & 575 & 625 & 529 \\
\hline 17 & 34 & 35 & 1190 & 1156 & 1225 \\
\hline 18 & 35 & 40 & 1400 & 1225 & 1600 \\
\hline 19 & 40 & 38 & 1520 & 1600 & 1444 \\
\hline 20 & 26 & 24 & 624 & 676 & 576 \\
\hline 21 & 28 & 25 & 700 & 784 & 625 \\
\hline 22 & 34 & 30 & 1020 & 1156 & 900 \\
\hline 23 & 26 & 29 & 754 & 676 & 841 \\
\hline 24 & 34 & 38 & 1292 & 1156 & 1444 \\
\hline 25 & 40 & 43 & 1720 & 1600 & 1849 \\
\hline 26 & 32 & 34 & 1088 & 1024 & 1156 \\
\hline 27 & 35 & 34 & 1190 & 1225 & 1156 \\
\hline 28 & 32 & 29 & 928 & 1024 & 841 \\
\hline 29 & 30 & 32 & 960 & 900 & 1024 \\
\hline 30 & 28 & 25 & 700 & 784 & 625 \\
\hline 31 & 24 & 26 & 624 & 576 & 676 \\
\hline 32 & 33 & 30 & 990 & 1089 & 900 \\
\hline 33 & 25 & 25 & 625 & 625 & 625 \\
\hline 34 & 34 & 39 & 1326 & 1156 & 1521 \\
\hline 35 & 33 & 29 & 957 & 1089 & 941 \\
\hline 36 & 40 & 35 & 1400 & 1600 & 1225 \\
\hline 37 & 38 & 42 & 1596 & 1444 & 1764 \\
\hline 38 & 40 & 42 & 1680 & 1600 & 1764 \\
\hline 39 & 36 & 31 & 1116 & 1296 & 961 \\
\hline 40 & 25 & 30 & 750 & 625 & 900 \\
\hline 41 & 28 & 26 & 728 & 784 & 676 \\
\hline 42 & 17 & 20 & 340 & 289 & 400 \\
\hline \multirow[t]{2}{*}{$\mathrm{N}=40$} & 13417 & 1351 & $\sum X Y$ & $\sum \times 2$ & $\Sigma Y 2$ \\
\hline & $\bar{X}=31,9$ & $\bar{Y}=32,2$ & 44407 & 44189 & 45099 \\
\hline
\end{tabular}

Fuente: Resultados obtenidos con el Test de habilidades $A$ y prueba de comprobación A. 
A partir de los resultados de la Tabla 1, se determinó la media aritmética de la variable $X 1$, y la variable $Y 1$, asimismo para ambas variables se extrajo la desviación típica y finalmente se aplicó la fórmula de coeficiente de correlación de Pearson para datos sin agrupar, de la siguiente manera:

$$
r_{X Y} \frac{\frac{\sum X Y}{N}-X Y}{S_{X} S_{Y}}=\frac{\frac{44407}{42}-(31,9)(32,2)}{(5,9)(6,1)}=0,837
$$

Interpretación:

Dado que el coeficiente de correlación de Pearson para datos simples es de $r=0,837$, este coeficiente se interpreta que existe una correlación positiva alta entre la Comprensión del problema (X1) y Promedio de notas obtenidas en las evaluaciones ( $Y 1)$, de modo que a mayor nivel de comprensión del problema, mayores promedios de notas obtenidas en matemática.

Sobre la relación entre el nivel de capacidad para elaborar un plan (X2) y el nivel de logro de competencias matemáticas (Y2) de los estudiantes del primer año de la especialidad de matemática de la Universidad Nacional "San Luis Gonzaga" de Ica, se tiene el siguiente cuadro:

Tabla 2

Relación entre el nivel de capacidad para elaborar un plan (X2) y el nivel de logro de competencias matemáticas (Y2) de los estudiantes.

\begin{tabular}{|c|c|c|c|c|c|}
\hline Muestra & $\hat{\jmath}$ & $Y$ & $X . Y$ & $x^{2}$ & $\mathrm{Y}^{2}$ \\
\hline 1 & 33 & 30 & 990 & 1089 & 900 \\
\hline 2 & 25 & 25 & 625 & 625 & 625 \\
\hline 3 & 34 & 39 & 1326 & 1156 & 1521 \\
\hline 4 & 33 & 29 & 957 & 1089 & 841 \\
\hline 5 & 40 & 35 & 1400 & 1600 & 1225 \\
\hline 6 & 22 & 20 & 440 & 484 & 400 \\
\hline 7 & 18 & 29 & 522 & 324 & 841 \\
\hline 8 & 22 & 25 & 550 & 484 & 625 \\
\hline 9 & 34 & 32 & 1088 & 1156 & 1024 \\
\hline 10 & 40 & 34 & 1360 & 1600 & 1156 \\
\hline 11 & 28 & 26 & 728 & 784 & 676 \\
\hline 12 & 33 & 30 & 990 & 1089 & 900 \\
\hline 13 & 25 & 25 & 625 & 625 & 625 \\
\hline 14 & 34 & 39 & 1326 & 1156 & 1521 \\
\hline 15 & 33 & 29 & 957 & 1089 & 841 \\
\hline 16 & 40 & 35 & 1400 & 1600 & 1225 \\
\hline 17 & 38 & 42 & 1596 & 1444 & 1764 \\
\hline 18 & 40 & 42 & 1680 & 1600 & 1764 \\
\hline 19 & 36 & 31 & 1116 & 1296 & 961 \\
\hline 20 & 25 & 30 & 750 & 625 & 900 \\
\hline 21 & 28 & 26 & 728 & 784 & 676 \\
\hline 22 & 17 & 20 & 340 & 289 & 400 \\
\hline 23 & 21 & 24 & 504 & 441 & 576 \\
\hline 24 & 27 & 32 & 864 & 729 & 1024 \\
\hline 25 & 22 & 20 & 440 & 484 & 400 \\
\hline 26 & 41 & 39 & 1599 & 1681 & 1521 \\
\hline 27 & 38 & 35 & 1330 & 1444 & 1225 \\
\hline 28 & 30 & 26 & 780 & 900 & 676 \\
\hline 29 & 22 & 24 & 528 & 484 & 576 \\
\hline 30 & 40 & 42 & 1680 & 1600 & 1764 \\
\hline 31 & 36 & 31 & 1116 & 1296 & 961 \\
\hline 32 & 25 & 30 & 750 & 625 & 900 \\
\hline 33 & 28 & 26 & 728 & 784 & 676 \\
\hline 34 & 17 & 20 & 340 & 289 & 400 \\
\hline 35 & 34 & 39 & 1326 & 1156 & 1521 \\
\hline 36 & 33 & 29 & 957 & 1089 & 841 \\
\hline 37 & 40 & 35 & 1400 & 1600 & 1225 \\
\hline 38 & 38 & 42 & 1596 & 1444 & 1764 \\
\hline 39 & 40 & 42 & 1680 & 1600 & 1764 \\
\hline 40 & 27 & 32 & 864 & 729 & 1024 \\
\hline 41 & 25 & 24 & 600 & 625 & 576 \\
\hline 42 & 30 & 32 & 960 & 900 & 1024 \\
\hline \multirow[t]{2}{*}{$\mathrm{N}=42$} & 1292 & 1297 & $\sum X Y$ & $\sum x^{2}$ & $\sum Y^{2}$ \\
\hline & $\bar{X}=30,8$ & $\bar{Y}=30,9$ & 41536 & 41888 & 41849 \\
\hline
\end{tabular}


A partir de los resultados de la tabla $\mathrm{N}^{\circ} 2$, se obtuvo la media aritmética de la variable $X 2$ que en este caso es de 30,8 puntos, de la variable $Y 2$ que es 30,9 puntos; asimismo la desviación estándar de X2 es de 6,9 puntos y de la variable Y2 es de 6,7puntos Con estos valores el coeficiente de correlación de Pearson se determinó con la siguiente formula:

$$
r_{X Y} \frac{\frac{\sum X Y}{N}-\bar{X} \bar{Y}}{S_{X} S_{Y}}=\frac{\frac{41536}{42}-(30,8)(30,9)}{(6,9)(6,5)}=0,830
$$

Interpretación

Este coeficiente de correlación de 0,830 , indica que existe una correlación alta entre la variable Capacidad para elaborar un plan (X2) y Nivel de logro de competencias matemáticas (Y2).

En consecuencia existe una relación positiva significativa entre el nivel de capacidad para elaborar un plan y el nivel de logro de competencias matemáticas de los estudiantes del primer año de la especialidad de matemática de la Universidad Nacional "San Luis Gonzaga" de Ica", puesto que el coeficiente de correlación fue $r=0,830$.

\section{Tabla 3}

Relación entre el nivel de desarrollo de la capacidad para ejecutar y evaluar un plan (X3) y el nivel de actitud positiva (Y3) de los estudiantes

\begin{tabular}{|c|c|c|c|c|c|}
\hline Muestra & $\hat{\mathbf{j}}$ & $Y$ & X.Y & $x^{2}$ & $\mathrm{Y}^{2}$ \\
\hline 1 & 23 & 20 & 460 & 529 & 400 \\
\hline 2 & 18 & 21 & 378 & 324 & 441 \\
\hline 3 & 32 & 34 & 1088 & 1024 & 1156 \\
\hline 4 & 35 & 34 & 1190 & 1225 & 1156 \\
\hline 5 & 32 & 29 & 928 & 1024 & 841 \\
\hline 6 & 41 & 43 & 1763 & 1681 & 1849 \\
\hline 7 & 20 & 25 & 500 & 400 & 625 \\
\hline 8 & 26 & 30 & 780 & 676 & 900 \\
\hline 9 & 30 & 25 & 750 & 900 & 625 \\
\hline 10 & 38 & 35 & 1330 & 1444 & 1225 \\
\hline 11 & 24 & 23 & 552 & 576 & 529 \\
\hline 12 & 23 & 20 & 460 & 529 & 400 \\
\hline 13 & 18 & 21 & 378 & 324 & 441 \\
\hline 14 & 32 & 34 & 1088 & 1024 & 1156 \\
\hline 15 & 35 & 34 & 1190 & 1225 & 1156 \\
\hline 16 & 32 & 29 & 928 & 1024 & 841 \\
\hline 17 & 30 & 32 & 960 & 900 & 1024 \\
\hline 18 & 28 & 25 & 700 & 784 & 625 \\
\hline 19 & 34 & 30 & 1020 & 1156 & 900 \\
\hline 20 & 26 & 29 & 754 & 676 & 841 \\
\hline 21 & 34 & 38 & 1292 & 1156 & 1444 \\
\hline 22 & 40 & 43 & 1720 & 1600 & 1849 \\
\hline 23 & 33 & 32 & 1056 & 1089 & 1024 \\
\hline 24 & 24 & 26 & 624 & 576 & 676 \\
\hline 25 & 25 & 23 & 575 & 625 & 529 \\
\hline 26 & 34 & 35 & 1190 & 1156 & 1225 \\
\hline 27 & 35 & 40 & 1400 & 1225 & 1600 \\
\hline 28 & 40 & 38 & 1520 & 1600 & 1444 \\
\hline 29 & 26 & 24 & 624 & 576 & 576 \\
\hline 30 & 28 & 25 & 700 & 784 & 625 \\
\hline 31 & 34 & 30 & 1020 & 1156 & 900 \\
\hline 32 & 26 & 29 & 754 & 676 & 841 \\
\hline 33 & 34 & 38 & 1292 & 1156 & 1444 \\
\hline 34 & 40 & 43 & 1720 & 1600 & 1849 \\
\hline 35 & 32 & 34 & 1088 & 1024 & 1156 \\
\hline 36 & 35 & 34 & 1190 & 1225 & 1156 \\
\hline 37 & 32 & 29 & 928 & 1024 & 841 \\
\hline 38 & 30 & 32 & 960 & 900 & 1024 \\
\hline 39 & 28 & 25 & 700 & 784 & 625 \\
\hline 40 & 24 & 26 & 624 & 576 & 676 \\
\hline 41 & 22 & 20 & 440 & 484 & 400 \\
\hline 42 & 35 & 33 & 1155 & 1225 & 1089 \\
\hline \multirow[t]{2}{*}{$N=42$} & 1268 & 1270 & $\sum X Y$ & $\sum X^{2}$ & $\sum Y^{2}$ \\
\hline & $\bar{X}=30,2$ & $\bar{Y}=30,2$ & 39769 & 39762 & 40124 \\
\hline
\end{tabular}

Fuente: Resultados obtenidos con el Test de habilidades C y prueba de comprobación C. 
Considerando los resultados de la Tabla 3, al aplicar las correspondientes fórmulas para determinar la media aritmética, la desviación estándar y el coeficiente de correlación de Pearson para datos sin agrupar se obtuvo los siguientes resultados: la media aritmética de la variable X3 fue 30,2 puntos; de la variable Y3 30,2 también.

En el caso de la desviación estándar, de la variable X3 es de 5,9 puntos, y de la variable $Y 3$ es de 6,6 puntos.

Reemplazando los valores para la determinación de coeficiente de correlación de Pearson se procesó de la siguiente manera:

$$
r_{X Y} \frac{\frac{\sum X Y}{N}-\bar{X} \bar{Y}}{S_{X} S_{Y}}=\frac{\frac{39769}{42}-(30,2)(30,2)}{(5,9)(6,6)}=0,895
$$

\section{Interpretación}

Según este resultado referente a la hipótesis específica 3 , el coeficiente de correlación es $r=0,895$ que indica que existe alta correlación positiva entre la variable Capacidad para ejecutar y evaluar el plan (X3) y la Actitud hacia las matemáticas (Y3).

En efecto, se evidencia que existe una relación positiva significativa entre el nivel de desarrollo de la capacidad para ejecutar y evaluar un plan y el nivel de actitud positiva de los estudiantes del primer año de la especialidad de matemática de la Universidad Nacional "San Luis Gonzaga" de Ica", dado que el coeficiente de correlación es de $r=0,895$

\section{DISCUSIÓN}

En esta investigación se reporta la existencia de una relación positiva entre la variable habilidades para la comprensión del problema mediante sus correspondientes dimensiones, y la variable promedio de notas obtenidos en las evaluaciones de los estudiantes del primer año de la especialidad de matemática de la Universidad Nacional "San Luis Gonzaga" de Ica; lo cual se verifica en el coeficiente de correlación de Pearson que resultó $r=0,837$.

Considerando las dimensiones de las variables generales como las habilidades para la comprensión del problema y el promedio de notas en matemática de los estudiantes de la muestra, se determina que existe una relación positiva alta entre la capacidad para elaborar un plan para la resolución de problemas y el nivel de logro de competencias matemáticas, entre la capacidad para ejecutar y evaluar el plan y la actitud hacia las matemáticas, y entre el nivel de desarrollo de las habilidades para la solución de problemas y el rendimiento académico en matemática de los estudiantes del primer año de la especialidad de matemática.

Estos coeficientes de correlación obtenidos, son similares a los que obtuvieron otros investigadores. Así, Herrera y Rodríguez (2011), en su investigación reportada en antecedentes sostiene que existe una relación significativa entre los estilos teórico y reflexivo con el rendimiento académico en matemáticas, y una relación inversa con los estilos activo y pragmático; del mismo modo la investigación realizada por Cerda (2014), sobre el Impacto de la resolución de problemas en el rendimiento académico de Matemática, reconoce que el empleo de la resolución de problemas como estrategia didáctica impactó de manera positiva en el rendimiento de los estudiantes.

En el ámbito nacional, Amarillo y Ventura (2012), mostraron en su investigación que existe una correlación mediana entre los Estilos de Aprendizaje y el Rendimiento Académico, lo cual se evidencia en un coeficiente de correlación que en este caso fue de $r=$ 0,683 .

Ante estos resultados concluye que el desarrollo de las habilidades para la resolución de problemas se relaciona significativamente con el rendimiento académico en matemática de los estudiantes. Las habilidades para la resolución de problemas se refieren a la capacidad de una persona para comprender, analizar, deducir, inducir, ejecutar, recopilar información, así como tener una actitud positiva frente a las dificultades que se presenten, constituyen sin duda condiciones que favorecen la resolución de los problemas no solamente de matemática sino de la vida.

\section{AGRADECIMIENTO}

Mi sincero agradecimiento a los catedráticos de la escuela de Postgrado de la Universidad Nacional Mayor de San Marcos y en especial a mi Asesor Dr. Fidel Chauca por su aporte invalorable en la materialización de esta investigación a Luhana y Jimena por ser el símbolo de mí Vida.

\section{REFERENCIAS BIBLIOGRÁFICAS}

Amarillo, H. J.E. y Ventura, C.E. (2014). Relación entre los estilos de aprendizaje y el Rendimiento Académico de los estudiantes de la especialidad de geografía de la facultad de Ciencias sociales y Humanidades de La Universidad Nacional de Educación Enrique Guzmán y Valle, 2012. Tesis para optar el título profesional de Educación. Universidad Nacional de Educación. Enrique Guzmán y Valle.

Batley, Tom (1991) Técnicas de gestión para profesionales. Cómo proyectar, organizar, dirigir y controlar para lograr resultados decididamente positivos, Barcelona, Granica.

Cerda, R.S.I.(2014), Impacto de la resolución de problemas en el rendimiento académico en intermedios. Tesis de maestría. Universidad Autonoma de Nueva León. México.

Ferrer, M. (2000). La Resolución de problemas en la restructuración de un sistema de habilidadesmatemáticas en la Escuela Media de Cuba. Tesis para optar el grado científico de Doctor en ciencias Pedagógicas. Instituto Superior Pedagógico "Frank Paes García". Facultad de Ciencias.

Fustier, M. (1989) La resolución de problemas, Francia, ESF Éditeur.

Gaulin, Claude (2001). Tendencias actuales en la resolución de problemas. Revista SIGMA, n. ${ }^{\circ} 19$. Bilbao. 
Herrera, N.y Rodriguez, J. (2011). Estilos de aprendizaje de los estudiantes de la Corporación Universitaria Aventista de Colombia y su Relación con el rendimiento Académico en el Area de Matemáticas. Revista Estilos de Aprendizaje. 4(7).124 - 139.

Polya, G., 1945. How to solve it. (Princeton University Press: Nueva Jersey). Trad. española: 1965, Cómo planteary resolver problemas. (Trillas: México). 